\title{
Brunnenbau in Entwicklungsländern. Problematik der Technik am Beispiel Äthiopiens
}

Aethiopien zählt heute zu den ärmsten - oder vielleicht besser ausgedrückt - zu den am wenigsten entwickelten Ländern der Erde. Dies kommt unter anderem auch darin deutlich zum Ausdruck, dass für etwa 75 o der Bevölkerung die Versorgung mit Trinkwasser ungenügend ist. Das Bestreben der Regierung, dieser Situation möglichst rasch und erfolgreich zu begegnen, ist somit verständlich. Dies stellt allerdings bei der Grösse des Landes mit einer Fläche von 1,22 Mio. $\mathrm{km}^{2}$ und einer Gesamtbevölkerung von 28,7 Mio. Menschen (1976) keine einfache Aufgabe dar.

Genauso wie in anderen Ländern der Dritten Welt mit ähnlichen Gegebenheiten wird auch in Aethiopien versucht, mit Hilfe moderner Technologien, in diesem Fall mittels Bohrmaschinen, dem Problem Trinkwasser wirkungsvoll $\mathrm{zu}$ begegnen.

Anhand von praktischen Erfahrungen werden die im Zusammenhang mit dem Einsatz von Bohrmaschinen auftretenden Schwierigkeiten aufgezeigt und mögliche Alternativen skizziert.

\section{Einsatz von Bohrmaschinen}

In Aethiopien ist seit 1976 die Ethiopian Water Resources Agency (EWRA) zuständig für Wasser. Diese Regierungsste1le ist aus der im Jahre 1971 eingesetzten National Water Resources Commission (NWRC) hervorgegangen und trägt die Verantwortung für eine optimale Nutzung vorhandener Wasservorkommen. Sie befasst sich in erster Linie mit Vorabklärungen, Projektierungen, Ausführungen und Unterhalt von Wassergewinnungsanlagen für Trinkwasser und Bewässerung, Hydrometeorologie sowie Koordinierung und Ueberwachung der Tätigkeiten weiterer Regierungsstellen, Privater und Hilfsorganisationen auf dem Gebiet Wasser.

Im Bereich Trinkwasserversorgungen konzentrierte sich die NWRC anfänglich praktisch ausschliesslich auf den Bau von Bohrbrunnen, die mit Unterwasserpumpen und einfachen Reservoiren ausgerüstet wurden. Der Grund für dieses einseitig orientierte Entwicklungskonzept lag vor allem darin, dass grösster Wert auf qualitativ einwandfreies Wasser ge:legt wurde, aus persönlichen Neigungen von seiten der Berater und wegen der in die Bohrtechnik gesetzten hohen Erwartungen betreffend Faktor Zeit.

Im Jahr 1974 wurden in Aethiopien 600 Bohrbrunnen gezählt. Ein Jahr später waren es 650 . Von

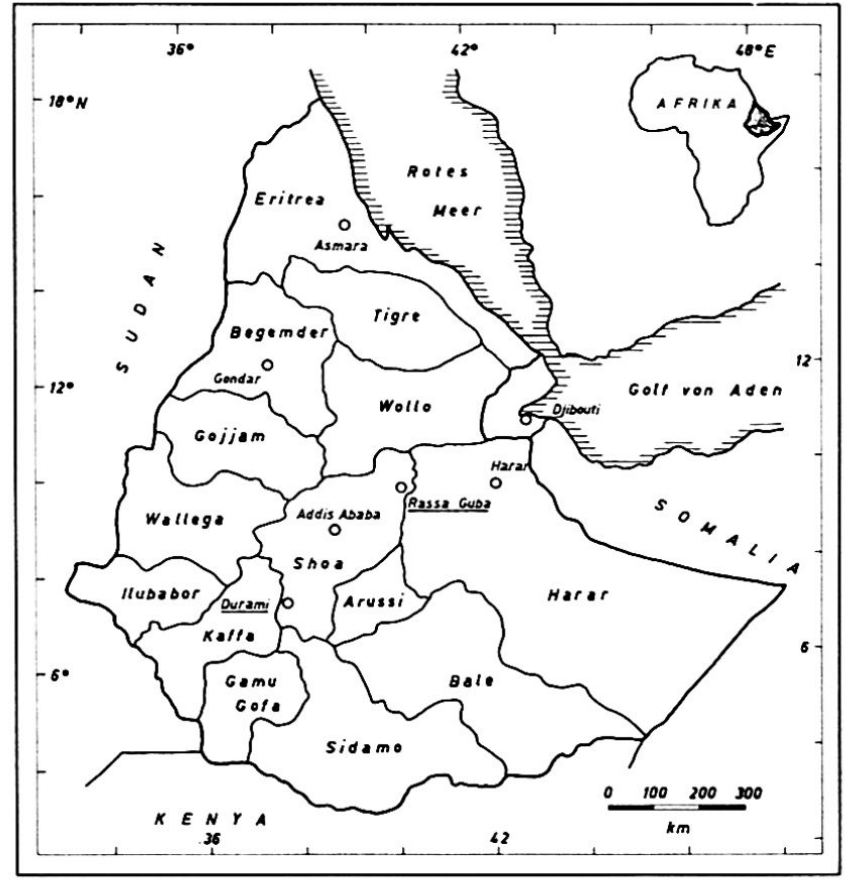

Abb.1: Aethiopien

zuständiger Seite wurde dazu bemerkt, dass der Bau dieser Brunnen viele Jahre in Anspruch genommen hätte, und dass bei einer Verdoppelung der Brunnenzahl das Problem Wasser erst für etwa 3 Mio. des insgesamt auf 21 Mio. Menschen geschätzten Bevölkerungsanteils mit mangelhafter Wasserversorgung einigermassen gelöst wäre.

Bei den rund 30 Bohrmaschinen, die zu Beginn des Jahres 1976 für die Beschaffung von Trinkwasser im Einsatz standen, handelte es sich je etwa zur Hälfte um Stauchbohrmaschinen (cable tool percussion drilling) und um Drehbohr- bzw. Schlagbohrmaschinen (core-, rotary-pneumatic percussion drilling). Zwei Bohrmaschinen wurden damals vom Ministry of Mines betreut, 18 von der ElWRA und zehn von Hilfsorganisationen. Der Einsatz der letzteren erfolgte im Zusammenhang mit der in den Jahren 1973/74 im Sahelgürtel ausgebrochenen Dürre- und Hungerkatastrophe, von der auch die äthiopischen Nordprovinzen Eritrea, Tigre, Wollo und Shoa betroffen waren (Abb.1).

Dr. Otto Langenegger, Emeligarten 375, 8592 Uttwil 
Einige Daten zu den üblichen Bohrbrunnen im Festgestein

Tiefe:

Durchmesser :

Verrohrung:

Pumpen:

\section{Brunnenertrag:}

Zeitaufwand für eine $100 \mathrm{~m}$ Bohrung:

\section{bis $150 \mathrm{~m}$}

5 bis 16 Zoll (12,5 bis $40 \mathrm{~cm})$

Stahl- und Kunststoffrohr

Handpumpen, vereinzelt mit Windmühlen betrieben;

elektrische Unterwasserpumpen (Stromerzeugung meist mittels Benzin- oder Dieselgeneratoren)

\section{0 bis $3001 / \mathrm{min}$.}

2 Tage bis mehrere Monate

Die EWRA rechnete in den Jahren 1975/76 mit einer durchschnittlichen Leistung von zwei bis drei Brunnen pro Bohrmaschine und Jahr. Die Kosten für einen solchen Bohrbrunnen einschliesslich Pumpe, Generator und Wassertank beliefen sich damals auf 50'000 bis 100'000 E\$ bei jährlichen Aufwendungen von 10'000 bis 15'000 E\$ für den Unterhalt (ungefährer Kurs: 1 ES $=1,30 \mathrm{Fr}$.).

Das Bohrteam einer Hilfsorganisation hatte von 1974 bis 1976 mit Hilfe einer für hartes Festgestein spezialisierten Bohrmaschine insgesamt 49 Bohrlöcher abgeteuft bis in maximale Tiefen von $115 \mathrm{~m}$ (Abb.2). Von diesen Bohrlöchern konnten 15 als Brunnen ausgebaut werden (Abb.3).

Die Erfolgsquote mit etwa $30 \%$ scheint gering zu sein. Wenn aber berücksichtigt wird, dass rund ein Drittel aller Bohrungen aus technischen Gründen aufgegeben werden musste, bevor die maximale Tiefe erreicht worden war, und dass vereinzelt wasserfündige Bohrlöcher wegen schlechter Wasserqualität nicht ausgebaut wurden, dann sieht die Sache wesentlich anders aus. Zudem hatte die Bohrmannschaft in der genannten Zeitspanne monatelange Unterbrüche auf sich zu nehmen infolge Anlaufschwierigkeiten, technischer Ausfälle und Nachschubproblemen. Ferner ist zu bemerken, dass in Zweifelsfällen betreffend Erfolgsaussicht, vor allem an Orten mit extrem schlechten Wasserverhältnissen, ein grösseres Risiko in Kauf genommen wurde als anderswo, denn es ging ja dabei nicht in erster Linie um die Wirtschaftlichkeit, sondern um Hilfeleistung.

Abgesehen von der Tiefe und der beim Bau angewandten Technik unterscheidet sich ein Bohrbrunnen von einem handgegrabenen Brunnen vor allem darin, dass eine Wasserentnahme nur bei Verwendung einer Pumpe möglich ist. Eine einfachere Methode, z.B. mit Hilfe eines Kübels, kommt wegen des kleinen Brunnendurchmessers nicht in Frage.

Diese Tatsache hat nun aber zur Folge, dass es sich bei einem Bohrbrunnen nicht nur im eine einmalige Anwendung der Technik in Form des Einsatzes einer Bohrmaschine handelt, sondern dass daraus ein Dauereinsatz technischer Mittel
(Pumpen) hervorgeht. Selbst die einfachste Handpumpe bedarf aber einer regelmässigen Wartung, und diese stellt erfahrungsgemäss den schwächsten Punkt dar innerhalb eines Brunnenbohrprojektes.

Eine in den Provinzen Tigre und Wollo im Jahre 1974 durchgeführte Erhebung zeigte, dass von insgesamt 30 Bohrbrunnen nur deren 10 betriebsbereit waren. Aehnliche Resultate sind aus Indien bekannt. Im Zusammenhang mit der grossen Bihar Hungersnot im Jahre 1966/67 wurden Tausende von Brunnen gebohrt und mit Handpumpen ausgerüstet. Acht Jahre später waren nur noch $40 \%$ davon in Funktion.

Welches sind nun aber die Ursachen für diesen Sachverhalt? Am Beispiel Aethiopien ist zunächst einmal festzustellen, dass ein grosser Mangel an technisch geschultem Personal herrscht. Ferner ist es aufgrund der gegebenen Organisations-, Klima- und Strassenverhältnisse vorläufig kaum möglich, für die über das ganze Land zerstreuten Brunnen einen zufriedenstellenden Unterhalt zu gewährleisten.

Um allen technischen Ausfällen gewachsen zu sein, muss ein Service-Team, abgesehen von Ersatzteilen und Werkzeugen einschliesslich Schweissapparatur, ausgerüstet sein mit einer Hebevorrichtung, beispielsweise in Form eines Dreifusses mit Winde oder Flaschenzug, um die üblicherweise an 2 Zoll Eisenrohren befestigten Pumpen aus bis über $100 \mathrm{~m}$ tiefen Brunnen zu heben. Der Transport einer solchen Ausrüstung verlangt aber ein entsprechendes Transportmittel. Ein Landrover kann solchen Anforderungen in vielen Fällen nur knapp gerecht werden.

Gut eingerichtete Hilfsorganisationen haben in der Regel nebst den Bohrequipen Teams im Einsatz, die sich mit dem Testen der Brunnen, den Pumpinstallationen und dem Service der Anlagen befassen. Solange diese Organisationen in ihren Einsatzgebieten tätig sind, funktioniert der Unterhalt im allgemeinen gut. Sobald aber das Feld geräumt wird, machen sich Schwierigkeiten bemerkbar. Selbstverständlich wird versucht, geeignete Lösungen zu finden. Ueblicherweise wird in solchen Situationen sämtliches Material, das für die Wartung von Brunnen erforderlich ist, der Regierung (EWRA) übergeben, aber wie bereits erwähnt, ist diese noch im Aufbau begriffene Stelle vorläufig nicht in der Lage, die gewünschte Kontinuität aufrechtzuerhalten.

Die ganze Problematik technischer Hilfe wird in einem Lande wie Aethiopien, das zur Zeit politisch derart unruhig ist, in dem Masse verstärkt, dass derartige Hilfeleistungen zum Teil sogar fragwürdig werden. Die dadurch bedingten Schwierigkeiten zeigen sich vor allem in den Bereichen Nachschub von Treibstoff, Material, Ersatzteilen und Lebensmitteln, Sicherheit von Personen und Sachen sowie im Umgang mit Behörden.

Zusätzlich zu den bereits erwähnten Problemen sind im Zusanmenhang mit Bohrbunnen vor allem auch die folgenden Punkte zu berücksichtigen: 


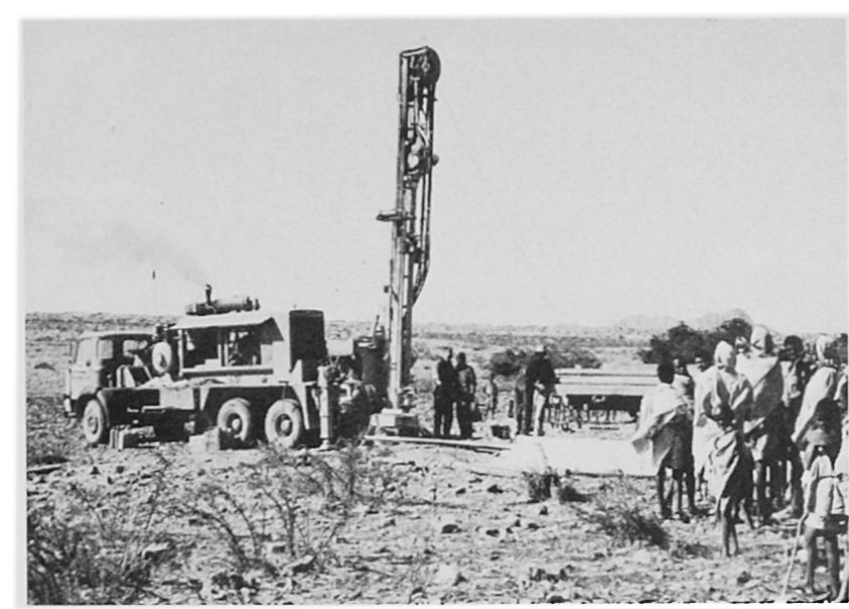

Abb.2: Einsatz einer Bohrmaschine in einer Höhe von 2200müM im Hochland der Tigre Provinz.

- Der Einsatz von Bohrmaschinen ist beschränkt auf Gebiete, die verkehrsmässig einigermassen erschlossen sind.

Dieser Faktor trifft insbesondere im Hochland von Aethiopien krass in Erscheinung, das wegen seiner teils stark gegliederten Gebirgslandschaft alles andere als verkehrsfreundlich ist.

- Unter den in Aethiopien herrschenden extremen klimatischen und topographischen Verhältnissen sind technische Ausfälle der Bohrmaschinen recht häufig. Reparatur- und Servicearbeiten sind aber sehr erschwert, weil Spezialisten meist fehlen und Ersatzteile aus dem Ausland beschafft werden müssen.

- Tiefengrundwasser ist qualitativ nicht immer geeignet als Trinkwasser. In Aethiopien sind es vor allem zu hohe Salz-, Nitrat- und Fluoridgehalte, die diesbezüglich als einschränkende Faktoren wirken.

- Bohren ist eine teure Angelegenheit. Es sind dabei nicht nur die hohen Anschaffungskosten für Maschinen und Geräte, Auslagen für deren

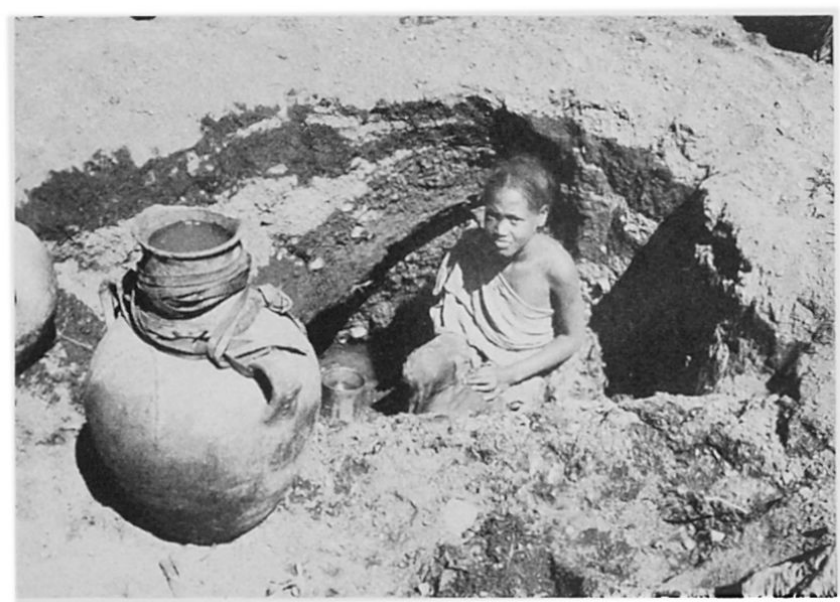

nivi.4: arockenfluss bei Maichare an der Ostabdachung zur Danakil Wüste (Rotes Meer), Tigre Provinz. In 50-100 cm Tiefe kommt im sandigkiesigen Flussbett sauberes Wasser zum Vorschein.

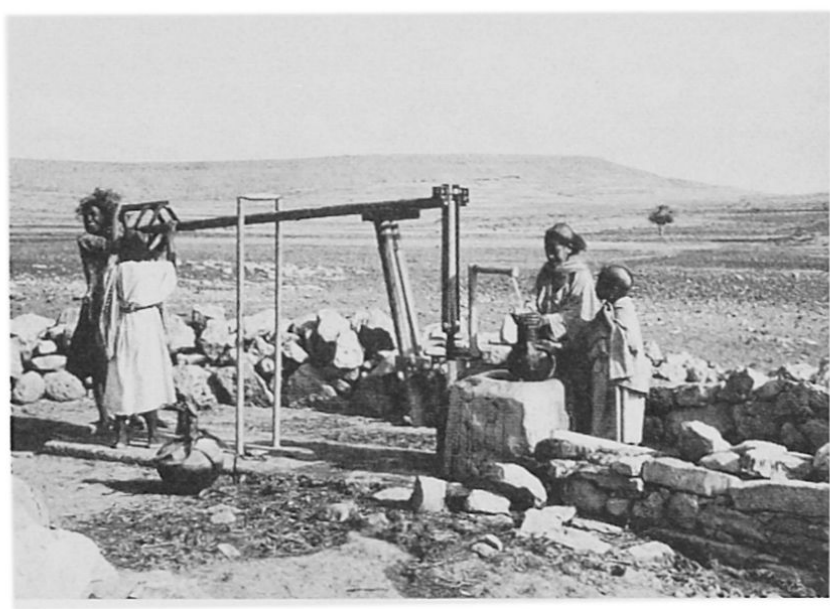

Abb.3: Mit Handpumpe ausgerüsteter Bohrorunnen in der Tigre Provinz.

Transport, Betrieb, Unterhalt und Bedienung, die ins Gewicht fallen, sondern auch die für den Brunnenbau erforderlichen Filterrohre und Pumpen.

- Bohrmaschinen sind in der Regel spezialisiert auf bestimmte geologische Verhältnisse und können somit im Einzeleinsatz aus hydrogeologischer Sicht selten optimal eingesetzt werden.

Eine Stauchbohrmaschine ist relativ einfach in der Handhabung und gut geeignet. für Lockergestein, nicht aber für hartes Festgestein. Demgegenüber stehen modernste Maschinen im Einsatz, mit denen in hartem Festgestein innert Stunden Tiefen von 50 und mehr Meter erbohrt werden können. Solche Maschinen werden aber vielfach dann problematisch, wenn in Lockergestein oder Festgestein mit weichen Wechsellagerungen gebohrt werden soll.

- Die seriöse Ausbildung von Einheimischen auf dem Fachgebiet Bohrtechnik ist unter den vorliegenden Gegebenheiten ein schwieriges und zeitaufwendiges Unterfangen und steckt noch ganz in den Anfängen.

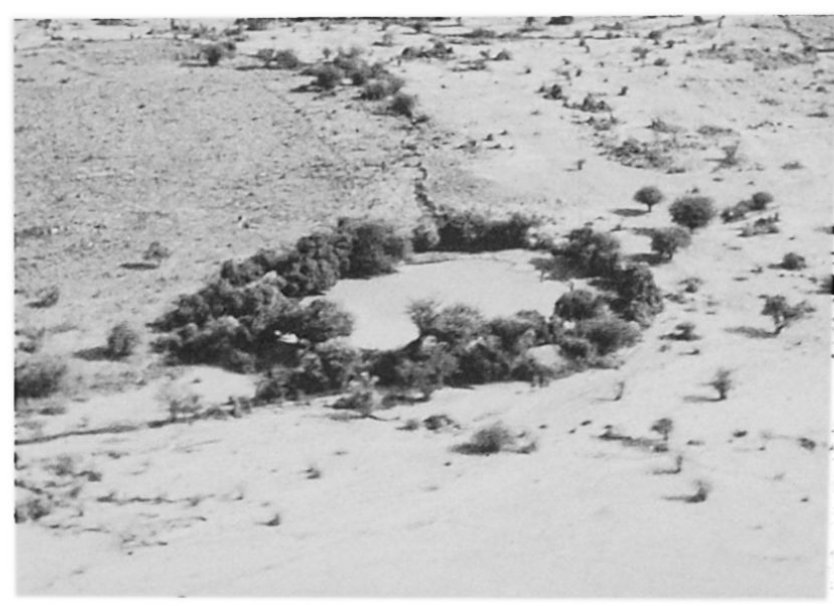

Abb.6: Einfacher Pond (Trinkwasserspeicher) im Gebiet der Western Tsamai im Süden der Gamu Gofa Provinz. 
Zusammenfassend kann somit gesagt werden, dass in Aethiopien der gezielte Einsatz von Bohrmaschinen für den Brunnenbau ein recht brauchbares Instrument darste11t. Aufgrund der vielen einschränkenden Faktoren kann dies jedoch für die grosse Masse der Landbevölkerung in absehbarer Zeit niemals die Antwort sein zum Problem Wasser, sondern lediglich ein Beitrag.

Was bisher am Beispiel der an sich phantastisch wirkenden Brunnenbohrtechnik aufgezeigt worden ist, kann weitgehend auf andere Gebiete der Entwicklungshilfe übertragen werden. Bei einem Bohrbrunnen tritt nun aber die ganze Problematik besonders krass in Erscheinung, weil ohne Technik (Pumpe) kein Wasser verfügbar gemacht werden kann, im Gegensatz etwa zu einer gefassten Quelle, deren Wasser mittels einer Leitung in freiem Gefälle zum Verbraucher gelangt und trotz möglicher Ausfälle von Hahnen oder Leitungsstükken weiterhin als Wasserlieferant funktionieren kann.

\section{Alternativen}

In Verbindung mit Fragen der Entwicklungshilfe taucht immer wieder die Gefahr auf, dass infolge der enormen kulturellen Unterschiede zwischen den sogenannten entwickelten und unterentwickelten Ländern die ganze Problemlage zu einseitig beurteilt wird. Der eigentliche Kern der Sache, der Mensch und seine Lebensweise, seine Kultur, vorwiegend durch naturgeographische Faktoren geprägt, wird zu wenig berücksichtigt.

Zur Sache Wasser drängt sich zum Beispiel die Frage auf, welche Bedeutung der Wassergüte in einem ersten Entwicklungsschritt überhaupt beigemessen werden muss. Die Wichtigkeit der Wasserqualität hinsichtlich Hygiene ist dem modernen Menschen zwar bekannt, nicht aber den breiten Volksschichten in Aethiopien. Als Illustration sei hier die in der Tigre Provinz zum Teil recht verbreitete Bevorzugung des Oberflächenwassers gegenüber dem Grundwasser erwähnt. Dies mag denn auch ein Grund dafür sein, dass die vielen von den Italienern während der Besetzungszeit (1936 - 1941) gegrabenen Brunnen zerstört oder zumindest nicht benützt worden sind.

Einwandfrei erstellte Bohrbrunnen bieten im allgemeinen Gewähr für bakteriologisch einwandfreies Wasser. Die Tatsache aber, dass das Wasser vom Wasserplatz zum Verbraucher transportiert werden muss, und zwar in der Regel auf hygienisch nicht einwandreie Art, sowie die Unkenntnis der hygienischen Grundprinzipien, vor allem im Ungang mit Lebensmitteln und Kleinkindern, stellt die Modellvorstellung der idealen Wasserversorgung im Sinne westlicher Kultur als erste Entwicklungsstufe in Frage. Es sei hier nur am Rande vermerkt, dass sich ja grundsätzlich qualitativ schlechtes Wasser mit Hilfe einfacher Mittel wie Abkochen, Filterung und entsprechender Aufbewahrung wesentlich verbessern lässt.
Von solchen Methoden wird nach wie vor beispielsweise auf Missionsstationen Gebrauch gemacht. Dass dem Glied Wasserstelle - Verbraucher eine nicht zu unterschätzende Bedeutung zukonmt, zeigen die in der folgenden Tabelle aufgeführten Angaben.

Prozentsatz der Todesfälle von Säuglingen im Alter bis vier Monate infolge Durchfallerkrankungen im Zusammenhang mit der Art der zur Verfügung stehenden Wasserversorgung.

\begin{tabular}{|c|c|}
\hline Art der Wasserversorgung & Todesfälle \\
\hline $\begin{array}{l}\text { Oeffentliche Wasserversorgung } \\
\text { mit Hausanschluss } \\
\text { ohne Hausanschluss, Distanz } \\
\text { Haus - Wasserhahn <100 m } \\
\text { ohne Hausanschluss, Distanz } \\
\text { Haus - Wasserhahn > } 100 \mathrm{~m} \\
\text { Ungeschützter Brunnen in nicht un- } \\
\text { mittelbarer Umgegung des Hauses }\end{array}$ & $\begin{array}{l}20,0 \% \\
57,1 \% \\
68,0 \% \\
57,6 \%\end{array}$ \\
\hline
\end{tabular}

Diese Angaben sind einer Veröffentlichung $z u$ einer vom Serviço Especial de Saude Publica, Rio de Janeiro, Brazil, im Jahre 1956 durchgeführten Untersuchung entnommen.

Diese Feststellungen lassen deutlich erkennen, welcher Stellenwert dem Menschen, seiner Einstellung und Aufklärung zur Sache beigemessen werden muss. Die Hilfe, in unserem Falle die Versorgung mit Wasser, genügt nicht. Erfolgreiche Aufklärungsarbeit ist aber in einem Volk wie die Aethiopier, das stolz und tradtionsgebunden ist, alles andere als einfach und erfordert viel Geduld und Zeit.

Aufgrund aller bisherigen Betrachtungen lässt sich dem Einsatz technischer Mittel für die Beschaffung von Trinkwasser folgende Alternative gegenüberstellen:

Einfacher, aber zweckmässiger und qualitativ einwandfreier Ausbau der von der Bevölkerung im grossen und ganzen bereits genutzten Wasservorkommen mit Hilfe einfacher Techniken und unter vollständiger Integration des Menschen mit seinem Wesen in den ganzen Entwicklungsprozess.

Hilfe bringen bedeutet somit in erster Linie, Impulse und Anregungen zur Selbsthilfe weitergeben, die Hauptarbeit, also etwa das Graben von Brunnen, muss jedoch von den Hilfsbedürftigen oder Hilfesuchenden selbst erbracht werden, damit eine richtige Beziehung zum Neuen entstehen kann. 
(sandiger Kies)

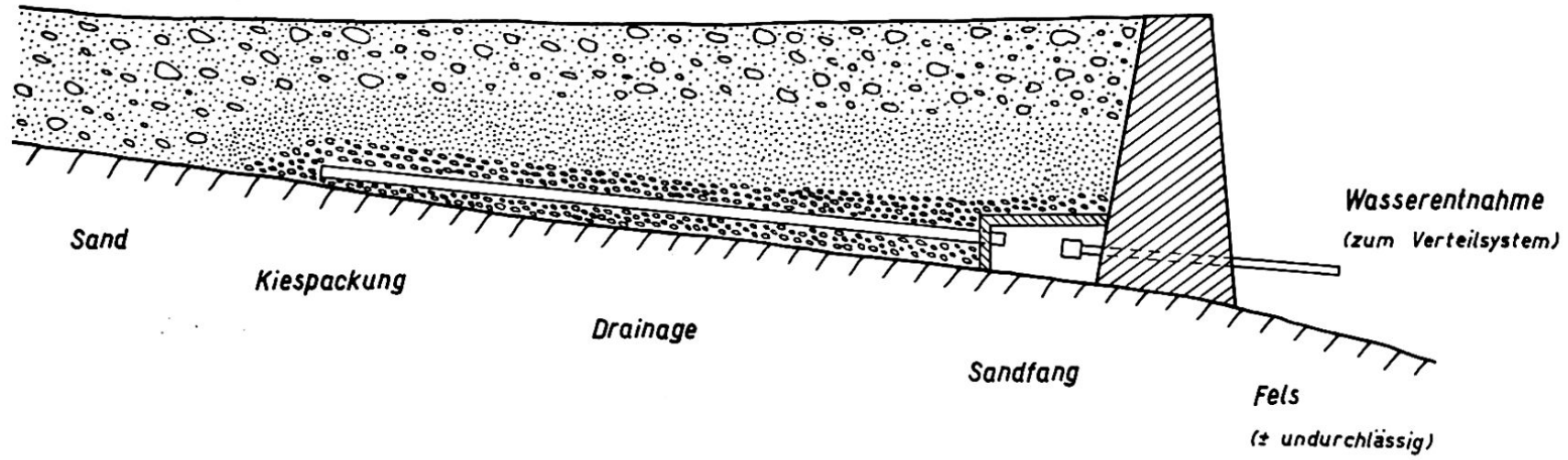

Abb.5: Projeitt Jurami. Prinzip der Bachwasserfassung mit eingebautem Sandfilter und Drainayesystem

Beim Ausbau von Wasserplätzen sind insbesondere drei Punkte zu berücksichtigen: der Schutz gegen Verunreinigungen, die Verwendung lokal vorhandener Baumaterialien und der Einbezug der Bevölkerung. Die dabei sich anbietenden Möglichkeiten umfassen Quellen, Grundwasser in Lockergesteinen und Flussbetten, Oberflächenwasser und Speicherung von Regenwasser. Bei Quellen geht es vor allem um das saubere Fassen und um den Bau angemessener Reservoire. Wenn man bedenkt, dass bei einem für äthiopische Landgebiete zutreffenden mittleren Wasserverbrauch von 5 Liter pro Kopf und Tag eine Quelle mit einer

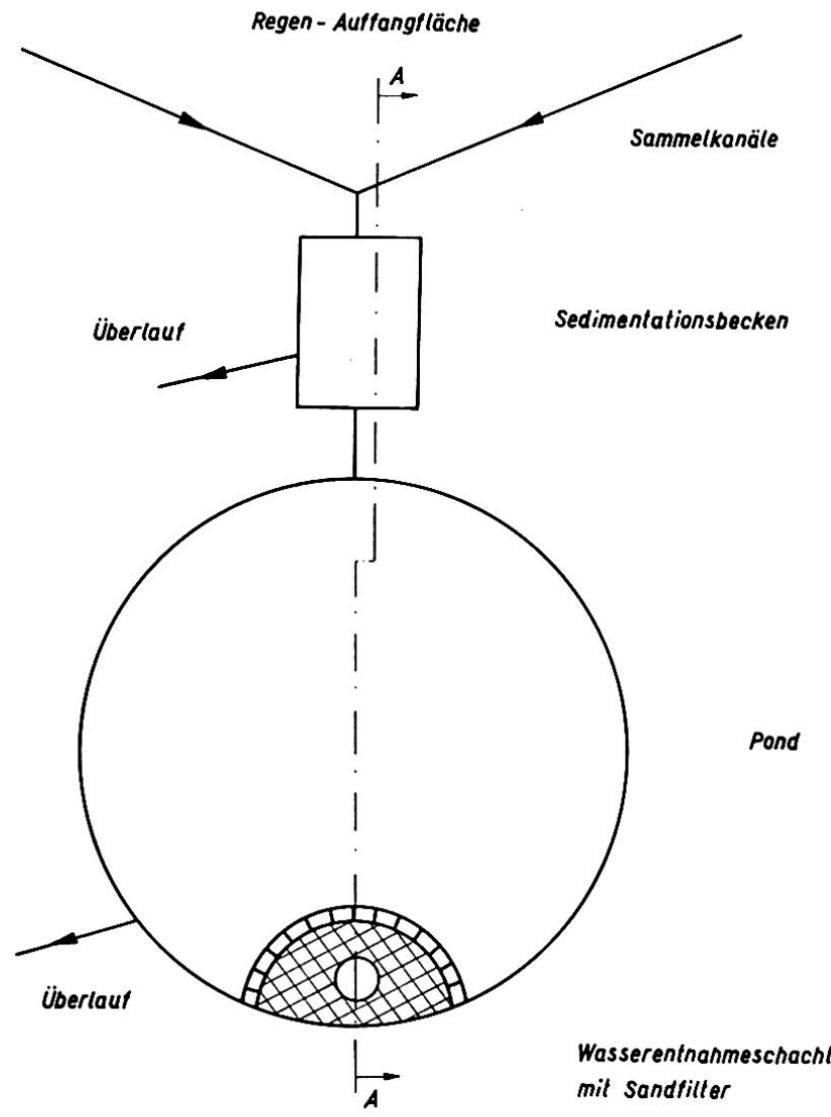

Abb.7: Projekt Rassa vuba. Prinzip eines Pondsystems bestehend aus wasserspeicher (Pond) mit Wasserentnahmevorrichtung u. Regenauffangfläche.
Schüttung von 1 Minutenliter ( 1 l/min.), bei optimaler Nutzung, d.h. unter Verwendung eines richtig bemessenen Reservoirs, ein Dorf mit einer Bevölkerung von 300 Einwohnern mit Wasser versorgen kann, dürfte es klar werden, welche Bedeutung den vielen im Hochland von Aethiopien vorhandenen Quellen zukommt.

Der Brunnenbau in Form von handgegrabenen Brunnen ist zwar nicht unbekannt, doch wird von dieser Möglichkeit zu wenig Gebrauch gemacht oder auf primitivste Art und Weise. Aus grundwasserführenden Betten von Trockenflüssen wird an vielen Orten Wasser entnommen, aber wiederum auf einfachste Art, nämlich mit Hilfe von ins Flussbett gegrabenen Löchern (Abb.4). Diese recht verbreiteten Wasservorkommen stellen vor allem in semiariden Gebieten wertvolle Nutzungsmöglichkeiten dar. Ein Beispiel dazu veranschaulicht das Projekt Durami (Abb.5). Wo Oberflächenwasser vorhanden ist, kann mit Hilfe von Sandfiltern eine gute Wasserqualität erreicht werden. Was die Speicherung von Regenwasser betrifft, findet man in Aethiopien einfache Ansätze (Abb.6), und zwar vorwiegend dort, wo dies für die Bevö1kerung die einzige Art der Wassergewinnung darstellt, abgesehen von stundenlangen Märschen zum nächsten wasserführenden Fluss. Es sind allerdings nur wenige Anlagen, die wenigstens von der Wassermenge her einigermassen zu genügen vermögen, d.h. nicht austrocknen. Diese Art der Wasserspeicherung ist übrigens sehr aktuell geworden in den USA und in Australien im Zusammenhang mit Farmen, allerdings unter Zuhilfenahme technischer Hilfsmittel zwecks Abdichtung des Untergrundes und Herabsetzung der Verdunstung.

Auch diese Methode der Wassergewinnung bietet vielversprechende Möglichkeiten, und zwar unabhängig von der Topographie. Die Abbildungen 7 und 8 zeigen das Grundprinzip eines Grundwasserspeichers (Pondsystem) und in Abbildung 9 sind Teilresultate einer Pondberechnung für das Dorf Rassa Guba graphisch dargestellt.

Sowohl für das Beispiel Durami (Abb.5) als auch für Rassa Guba (Abb.7-9) gilt, dass ganz beträchtliche Verbesserungen in bezug auf Wassermenge und Wassergüte erzielt werden können im 
Auffangfläche

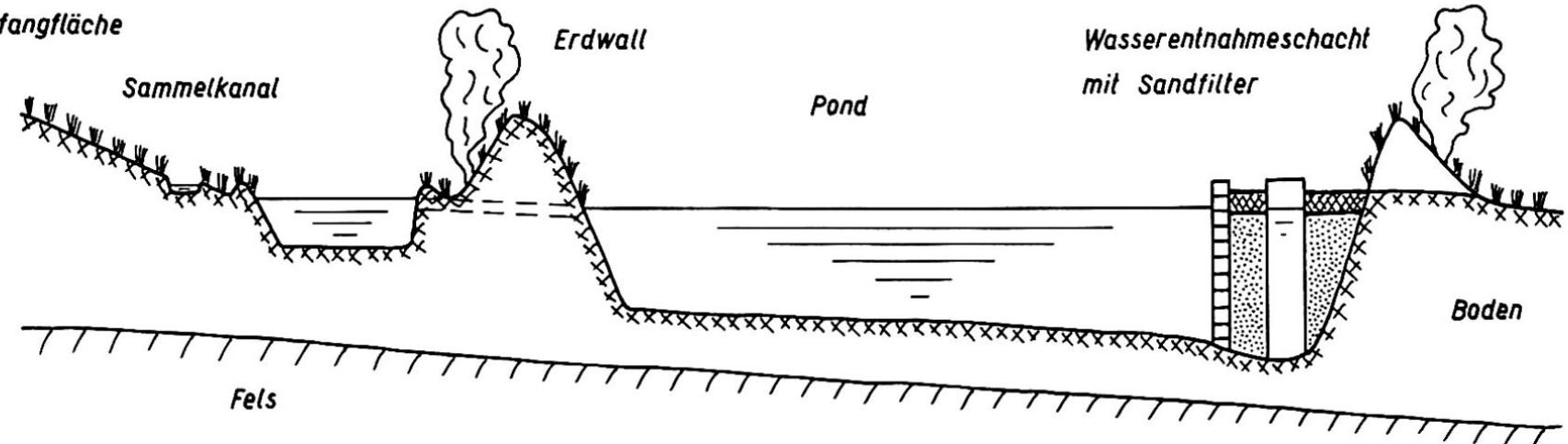

-Abb.8: Projekt Rassa Guba. Prinzip eines Pondsystems (Schnitt A - A ).

Vergleich zu dem, was diesbezüglich in Gebrauch ist. Zudem sind derartige Wasserversorgungen

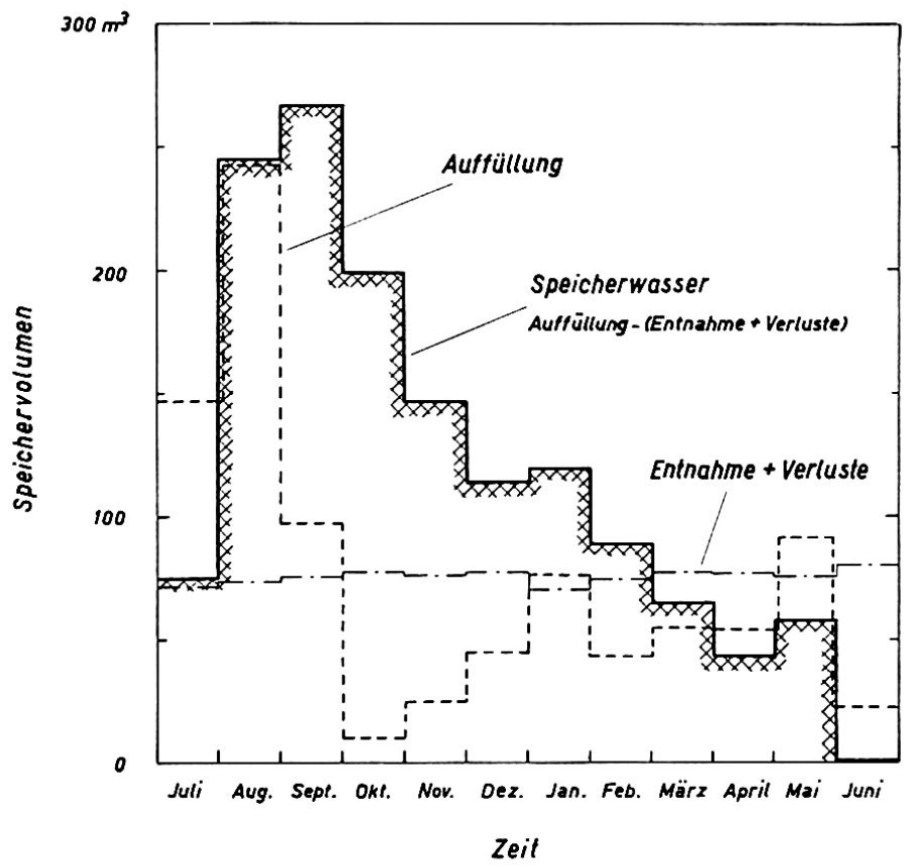

Abb.9: Projekt Rassa Guba. Darstellung der monatlichen Bilanz des Speicherwassers, der Auffüllung und der Entnahme + Verluste. schrittweise ausbaubar. Faszinierend an einem Pondsystem ist sicherlich die Tatsache, dass ein solches grundsätzlich mit einfachsten Werkzeugen, wie sie von der Landbevölkerung im Alltag gebraucht werden, und mit lokal verfügbaren Baustoffen realisierbar ist.

Das Projekt Durami wurde im Sommer 1977 erfolgreich abgeschlossen und stellt eine echte Alternativlösung dar zu erfolglos durchgeführten Bohrversuchen. In solchen Fällen, wo der Einsatz von Bohrmaschinen zu einem Misserfolg wird, bleibt schlussendlich doch nur eine Möglichkeit, um die Situation betreffend Trinkwasser zu verbessern, nämlich die Wahl der günstigsten Alternative. Gleichzeitig wird aber auch klar, welche Bedeutung dem Hydrologen/Hydrogeologen bei solchen Problemen zukommt, und zwar vor allem im Zusammenhang mit der Beschaffung von Grundlagenmaterial und der Vermittlung des know-how.

Das grosse Interesse, das die zuständigen Stellen der Ethiopian Water Resources Agency dem Durami Projekt entgegengebracht hatten, ist ein Hinweis dafür, dass der Anwendung einfacher Techniken in Bereich der Trinkwasserbeschaffung auch von seiten der ElWRA vermehrte Aufmerksamkeit geschenkt wird. Der Schlussatz in der Stellungnahme zur Projekteingabe lautete denn auch: "It is therefore recommended that the proposal should be approved, and that EWRA should maintain a continuing interest in this novel development." 